\title{
Theory and simulations of a gyrotron backward wave oscillator using a helical interaction waveguide
}

\author{
W. He, ${ }^{\text {a) }}$ A. W. Cross, A. D. R. Phelps, K. Ronald, and C. G. Whyte \\ SUPA, Department of Physics, University of Strathclyde, Glasgow G4 ONG, United Kingdom \\ S. V. Samsonov, V. L. Bratman, and G. G. Denisov \\ Institute of Applied Physics, Russian Academy of Sciences, Nizhny Novgorod 603950, Russia
}

(Received 11 May 2006; accepted 16 July 2006; published online 1 September 2006)

\begin{abstract}
A gyrotron backward wave oscillator (gyro-BWO) with a helically corrugated interaction waveguide demonstrated its potential as a powerful microwave source with high efficiency and a wide frequency tuning range. This letter presents the theory describing the dispersion properties of such a waveguide and the linear beam-wave interaction. Numerical simulation results using the PIC code MAGIC were found to be in excellent agreement with the output measured from a gyro-BWO experiment. (C) 2006 American Institute of Physics. [DOI: 10.1063/1.2345607]
\end{abstract}

The gyrotron backward wave oscillator (gyro-BWO) is an efficient source of frequency-tuneable high power coherent radiation. It is based on the resonant cyclotron interaction of electrons gyrating in the external magnetic field with an oppositely traveling electromagnetic wave. A number of gyro-BWOs have been investigated both in theory ${ }^{1-4}$ and experiments. $^{5-8}$ Two such experiments at the Naval Research Laboratory $^{5}$ and the National Tsing Hua University ${ }^{6}$ operating at the fundamental mode of a smooth cylindrical waveguide demonstrated excellent voltage tuning bandwidth of up to $5 \%$ and $13 \%$, respectively, with a high efficiency of nearly $20 \%$ at power levels of up to $100 \mathrm{~kW}$ at Ka-band frequencies.

A microwave system in the form of a helically corrugated waveguide has been demonstrated with a wave dispersion that has a near constant group velocity in the region of small axial wave number. ${ }^{9}$ This allows broadband microwave amplification to be achieved in a gyrotron traveling wave amplifier. ${ }^{10,11}$ Such a system is also favorable when used in a gyro-BWO (Ref. 12) in two ways. Firstly, due to its larger group velocity as compared with conventional gyroBWOs, it has a wider frequency tuning range. Secondly, due to the nonsymmetrical geometry of the helical interaction region, the electron beam interacts only with the wave in one direction (either backward or forward wave) and hence has a pure frequency component. Previous experiments using such a microwave system at $\mathrm{Ka}$ band achieved an output power of $\sim 1 \mathrm{MW}$, an efficiency of $10 \%$, and a frequency tuning band of $15 \%$ using a $20 \mathrm{~ns}, 300 \mathrm{keV}$ electron beam. ${ }^{13}$ Recently a relative frequency tuning band of $17 \%$ at $X$ band $^{14}$ with $16.5 \%$ electronic efficiency was achieved at the second harmonic of the electron cyclotron mode using a threefold helically corrugated waveguide and an axis-encircling electron beam. The dimensions of the helical structure used in this experiment were designed to support the operating eigenwave of a higher group velocity as compared with that used in Ref. 13 resulting in a higher electronic efficiency and wider frequency tuning range. In this letter the equations describing the dispersion property and linear wave interaction for this type of gyro-BWO are presented and applied to the latest experiment. ${ }^{14}$ In addition, simulation results of the

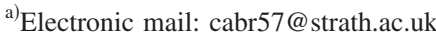

gyro-BWO interaction using a three-dimensional PIC code MAGIC (Ref. 15) as compared with the experimental measurements are presented.

The threefold helical waveguide structure can provide effective resonant coupling between two partial rotating waves of the waveguide, i.e., the first spatial harmonic of the traveling $\mathrm{TE}_{11}$ mode and the near cutoff $\mathrm{TE}_{21}$ mode when the period of the structure is properly chosen to meet the Bragg resonance conditions. The resonant coupling of the waves corresponds to the intersection of their dispersion curves (in Fig. 1). If the amplitude of the corrugation is small compared with the wavelength, the coupling equations for the two partial waves in the gyro-BWO interactions can be described approximately in the operating frequency region by the following equation:

$$
\begin{aligned}
& \frac{d^{2} f}{d z^{2}}+2 k_{0} \delta f=2 \sigma k_{0} b, \\
& i h_{0} \frac{d b}{d z}+\left(k_{0} \delta-h_{0} \Delta_{g}\right) b=\sigma k_{0} f,
\end{aligned}
$$

where $f$ and $b$ are the amplitudes of the near cutoff and traveling partial waves, respectively, $\sigma$ the coupling factor ${ }^{16}$ between them, $\delta$ and $\Delta_{g}$ the frequency and geometrical mismatch, and $k_{0}$ and $h_{0}$ the geometrical constants. ${ }^{9}$ One can therefore obtain the dispersion of the eigenwave in the gyroBWO interactions:

$$
\left(h^{2}-2 \delta\right)\left(h-\Delta_{g}+\delta / h_{0}\right)+2 \sigma^{2} / h_{0}=0 .
$$

One of the eigenwaves, i.e., $w_{1}$, having a near constant negative group velocity in the designed operating frequency range, is the operating eigenwave of the interaction. This operating eigenwave, resulting from the resonant scattering of the counter-rotating (with respect to the rotation of the helical waveguide) $\mathrm{TE}_{11}$ mode into the corotating $\mathrm{TE}_{21}$ mode inside the helical waveguide, is used in the experiment to resonantly interact with the second harmonic of the electron cyclotron mode as shown in Fig. 1.

The cyclotron resonance maser (CRM) instability provided the effective interaction between the radiation and the axis-encircling electron beam with the interaction harmonic number equal to the azimuthal mode index of the radiation. 


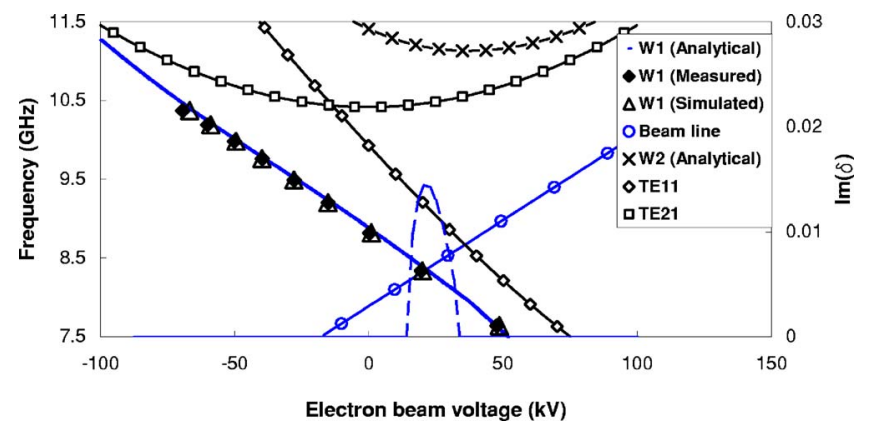

FIG. 1. Dispersion diagram of the operating eigenwave, the second harmonic electron cyclotron mode, and negative imaginary solution of $\delta$ from the gyro-BWO experiment.

In analogy to gyrotron interactions and Ref. 9, it is possible for one to derive the gyro-BWO beam-wave dispersion:

$$
\begin{gathered}
{\left[\left(h^{2}-2 \delta\right)\left(h-\Delta_{g}+\delta / h_{0}\right)+2 \sigma^{2} / h_{0}\right]\left[h-\left(\delta-\Delta_{H}\right) / \beta_{z 0}\right]^{2}} \\
\quad=C^{3}\left(h-\Delta_{g}+\delta / h_{0}\right)\left\{1+\frac{2 s}{\alpha_{0}^{2} \beta_{z 0}}\left[h-\frac{\left(\delta-\Delta_{H}\right)}{\beta_{z 0}}\right]\right\}
\end{gathered}
$$

where $\alpha_{0}$ and $\beta_{z 0}$ are the beam initial pitch angle and relative velocity in the longitudinal direction, respectively. The interaction frequency of the gyro-BWO can be calculated by solving the uncoupled beam-wave equation by setting $C=0$ in Eq. (3), i.e., the intersection of the eigenwave $w_{1}$ and the beam dispersion line. In a general case, Eq. (3) has four $\delta(h)$ roots, with two real roots being the "hot" (electron beam present) eigenwaves, and a pair of conjugate complex roots, which are degenerates of the electron cyclotron mode due to the CRM interaction at and near the intersection when the beam parameters are suitably chosen. The negative imaginary number of the solution (Fig. 1, dashed line showing one of such interaction for the gyro-BWO) gives rise to the oscillation that grows with time in the cavity and hence allows the starting condition and the small signal growth of the oscillation to be analyzed.

The dispersion of the operating eigenwave can be found by measuring the phase evolution of a counter-rotating circularly polarized wave when it propagates through the waveguide by using a vector network analyzer. ${ }^{17}$ It can also be measured by detecting the polarization angle of a linearly polarized wave when it propagates through the waveguide by using a scalar network analyzer (SNA). ${ }^{9,17}$ In Fig. 1 the measured results using the SNA method are shown and compared with the results simulated from MAGIC using the same oper-

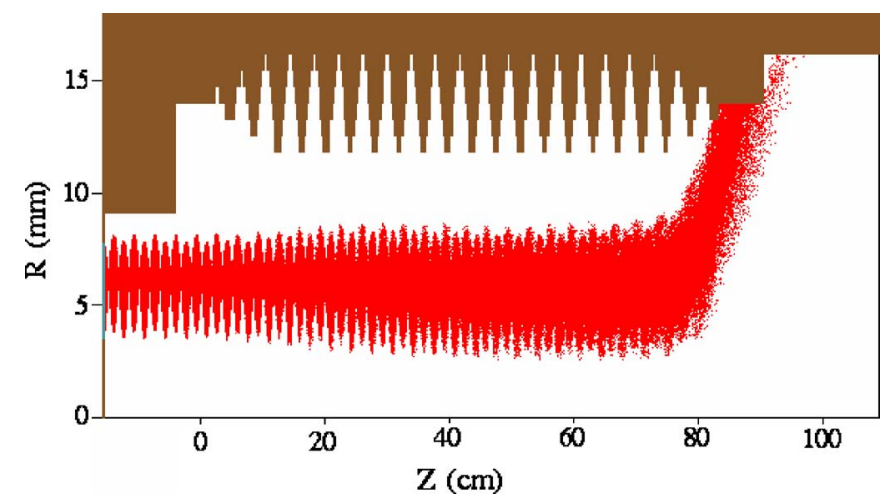

FIG. 2. Geometry of beam-wave interaction simulations by MAGIC and a snapshot of interacting beam trajectories.
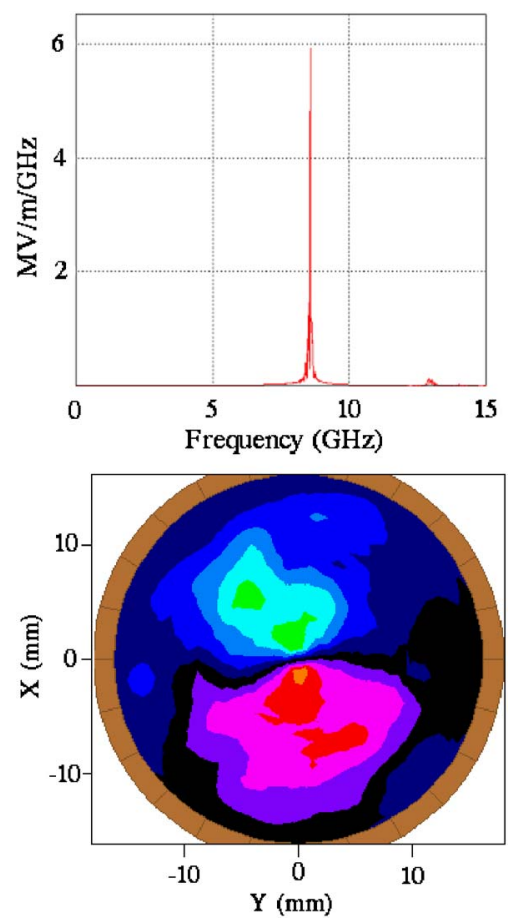

FIG. 3. MAGIC simulation result showing the output spectrum of $8.575 \mathrm{GHz}$ and mode pattern of $\mathrm{TE}_{11}$.

ating eigenwave. In the simulation using the MAGIC code, a left-polarized circular wave of one frequency was injected into the right-hand helical waveguide, and a component of the electric field inside the waveguide was measured along the axial direction. The measured field was then numerically analyzed and the axial wave number of the eigenwave was therefore obtained for that frequency.

The radiation of the gyro-BWO can be coupled out at two positions: one from an output coupler at the upstream side of the electron beam and the other through the output window at the downstream end. In the latter case the output window will act as a boundary of the cavity; therefore some reflection from the window (can be as low as 1\%) is desirable for the oscillation to start. Our experiments confirmed that the performance of the gyro-BWO is the same when using the two different output methods. In the simulation of the gyro-BWO, the simpler geometry where the output radiation was coupled out of the window was used to simulate the beam wave interaction as shown in Fig. 2. An electron beam with parameters similar to those measured in the experiment was used, i.e., beam energy, current, and beam pitch angle, guided by a magnetic field in a beam tube with a radius of $9 \mathrm{~mm}$ in which the lowest order mode was cut off and propagating in the downstream (right) direction. For the gyroBWO to oscillate, the electron beam should rotate in the opposite direction to the helical structure. At the downstream end, a small step-up (14-16 mm) in the output waveguide radius (instead of the output window) was used to define the interaction cavity. The step-up had a reflection coefficient of $5 \%-6 \%$ for the operating frequency range. In the simulation the emitted electron beam can either have an annular geometry or be in the form of a solid axis-encircling beam. The simulations showed that the radiation power and frequency are very similar using either type of beam shape as long as 


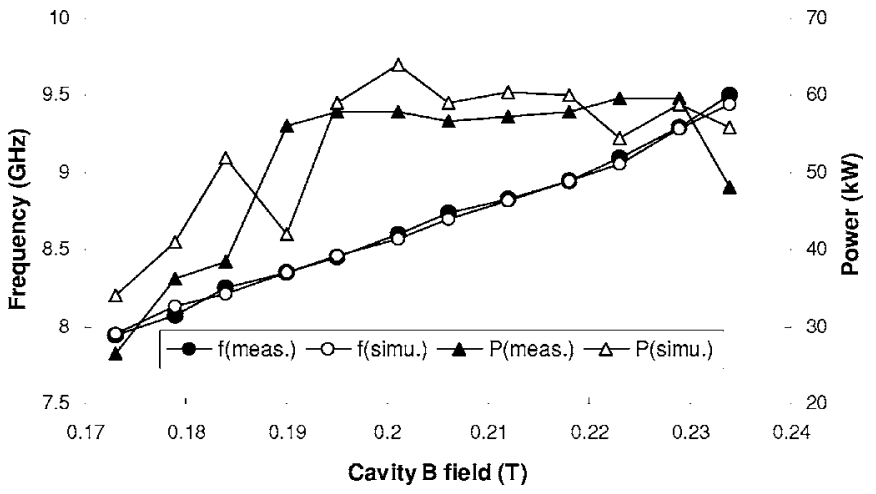

FIG. 4. Measured and simulated output frequency and power of the gyroBWO as a function of tuning cavity magnetic field.

the beam parameters are kept the same. A snapshot of the simulation showing the geometry and electron beam trajectories of an axis-encircling solid beam that was used in the experiment is shown in Fig. 2. When the magnetic field was $0.2008 \mathrm{~T}$, electron beam energy $185 \mathrm{keV}$, current $2 \mathrm{~A}$, and pitch alpha 1.1, a simulated output of $\sim 64 \mathrm{~kW}$ and frequency of $8.587 \mathrm{GHz}$ were obtained, which are very close to the output power of $58 \mathrm{~kW}$ and frequency of $8.58-8.60 \mathrm{GHz}$ measured in the experiment. A typical simulated output spectrum and mode pattern are shown in Fig. 3.

At beam energy of $185 \mathrm{keV}$ and current of $2 \mathrm{~A}$, the simulated output frequency and power from the gyro-BWO, as a function of the tuning cavity magnetic field, were compared with the measured results and are shown in Fig. 4. Also the frequency tuning characteristic of the gyro-BWO using beam voltage as a tuning parameter at a fixed cavity magnetic field of $0.195 \mathrm{~T}$ was simulated and is shown in Fig. 5. Figures 4 and 5 clearly show that the simulation results are in very good agreement with the measured results from the experiments. It should be noted that when a cavity magnetic field in excess of $0.240 \mathrm{~T}$ was applied, microwave radiation of similar power level was still generated. However, the frequency of the microwave radiation in such a case was measured to be around $10.5-10.6 \mathrm{GHz}$ and was predicted by MAGIC simulations, which corresponded to the normal gyrotron interaction, in this case, the forward wave second harmonic gyrotron interaction with the $\mathrm{TE}_{21}$ mode.

A frequency-tuneable gyro-BWO experiment using a helical waveguide was analyzed and simulated. The linear theory of the beam wave interaction for the gyro-BWO based on the helical waveguide would allow detailed analysis of starting current and growth rate of the oscillation. Excellent agreement was shown between the simulations of the gyroBWO using MAGIC and the measured results in the experiments.

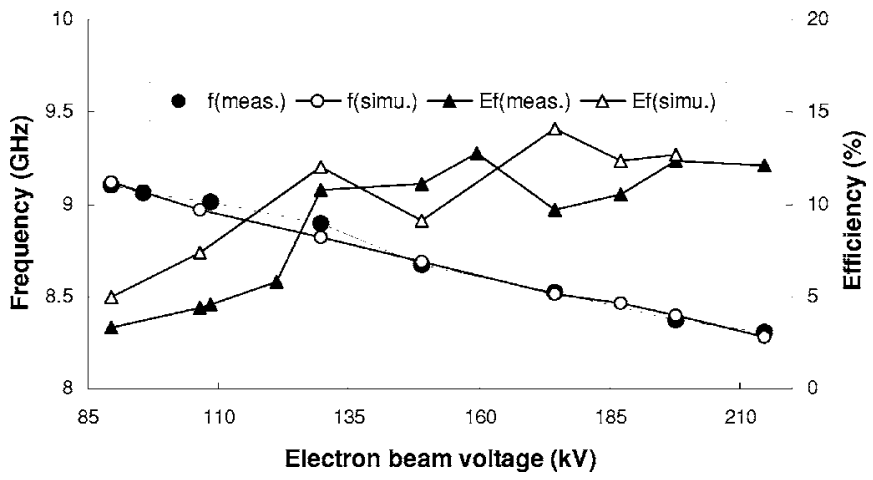

FIG. 5. Measured output frequency and interaction efficiency as a function of tuning e-beam voltage in comparison with the simulation.

This work was supported by the UK Engineering and Physical Sciences Research Council (EPSRC), DSTL, e2v, TMD, the UK Faraday Partnership in High Power Radio Frequency Engineering, Gycom Ltd., Nizhny Novgorod, Russia, and the Russian Foundation for Basic Research under Grant No. 01-01-16780.

${ }^{1}$ V. K. Yulpatov, Radiophys. Quantum Electron. 10, 471 (1967).

${ }^{2}$ N. S. Ginzburg, I. G. Zaritsyna, and G. S. Nusinovich, Radio Eng. Electron. Phys. 24, 113 (1979).

${ }^{3}$ A. K. Ganguly and S. Ahn, Int. J. Electron. 67, 261 (1989).

${ }^{4}$ G. S. Nusinovich and O. Dumbrajs, IEEE Trans. Plasma Sci. 24, 620 (1996).

${ }^{5}$ S. Y. Park, R. H. Kyser, C. M. Armstrong, R. K. Parker, and V. L. Granatstein, IEEE Trans. Plasma Sci. 18, 321 (1990).

${ }^{6}$ C. S. Kou, S. G. Chen, L. R. Barnett, H. Y. Chen, and K. R. Chu, Phys. Rev. Lett. 70, 924 (1993).

${ }^{7}$ T. A. Spencer, R. M. Gilgenbach, and J. J. Choi, J. Appl. Phys. 72, 1221 (1992).

${ }^{8}$ M. A. Basten, W. C. Guss, K. E. Kreischer, R. T. Temkin, and M. Caplan, Int. J. Infrared Millim. Waves 16, 889 (1995).

${ }^{9}$ G. G. Denisov, V. L. Bratman, A. D. R. Phelps, and S. V. Samsonov, IEEE Trans. Plasma Sci. 26, 508 (1998).

${ }^{10}$ G. G. Denisov, V. L. Bratman, A. W. Cross, W. He, A. D. R. Phelps, K. Ronald, S. V. Samsonov, and C. G. Whyte, Phys. Rev. Lett. 81, 5680 (1998).

${ }^{11}$ V. L. Bratman, A. W. Cross, G. G. Denisov, W. He, A. D. R. Phelps, K. Ronald, S. V. Samsonov, C. G. Whyte, and A. R. Young, Phys. Rev. Lett. 84, 2746 (2000).

${ }^{12}$ S. V. Samsonov, V. L. Bratman, G. G. Denisov, M. Yu. Glyavin, V. K. Lygin, A. G. Luchinin, Yu. V. Bykov, A. A. Bogdashov, and M. Thumm, IEEE Trans. Plasma Sci. 32, 884 (2004).

${ }^{13}$ V. L. Bratman, G. G. Denisov, V. N. Manuilov, S. V. Samsonov, and A. B. Volkov, in Digest of the 26th International Conference on Infrared and Millimeter Waves, Toulouse, France, 2001.

${ }^{14}$ W. He, K. Ronald, A. R. Young, A. W. Cross, A. D. R. Phelps, C. G. Whyte, E. G. Rafferty, J. Thomson, C. W. Robertson, D. C. Speirs, S. V. Samsonov, V. L. Bratman, and G. G. Denisov, IEEE Trans. Electron Devices 52, 839 (2005).

${ }^{15}$ T. Gray, D. N. Smithe, and Larry D. Ludeking, Introduction to MAGIC (Mission Research, Newington, VA, 2003).

${ }^{16}$ S. J. Cooke and G. G. Denisov, IEEE Trans. Plasma Sci. 26, 519 (1998).

${ }^{17}$ G. Burt, K. Ronald, A. D. R. Phelps, A. W. Cross, I. V. Konoplev, W. He, A. R. Young, C. G. Whyte, and J. Thomson, Phys. Rev. E 70, 046402 (2004). 